\title{
The Inter-Galactic Populations and Unbound Dark Matter
}

\author{
Ing-Guey Jiang ${ }^{* *}$ \\ Department of Physics and Institute of Astronomy, \\ National Tsing-Hua University, Hsin-Chu City, Taiwan \\ E-mail: jiang@phys.nthu.edu.tw
}

\section{Yu-Ting Wu}

Department of Physics, National Tsing-Hua University, Hsin-Chu City, Taiwan

E-mail: d9622814doz.nthu.edu.tw

\begin{abstract}
The diffused light between galaxies, as first discovered by Zwicky (1951), has been an interesting issue related with the evolution of galaxies. N-body simulations of mergers of galaxies with dark matter have been employed to address this topic. By N-body simulations, we investigate the production of intergalactic populations through head-on mergers. Our results show that single head-on mergers cannot explain the observations, and the main conclusions are: (1)head-on merging events make a considerable fraction of dark metter become unbound; (2)some luminous intergalactic populations are in fact trapped in dark halo and gravitationally bounded; (3)multiple and minor mergers shall have contributions on intergalactic populations.
\end{abstract}

Identification of Dark Matter 2010-IDM2010

July 26-30, 2010

Montpellier France

\footnotetext{
* Speaker.

${ }^{\dagger}$ We are grateful to the National Center for High-performance Computing for computer time and facilities. This work is supported in part by the National Science Council, Taiwan, under NSC 98-2112-M-007-006-MY2.
} 


\section{Introduction}

The origin of the diffused light between galaxies, as first discovered by Zwicky (1951) in the Coma cluster, has been a long term puzzle because in the standard picture, stars in the universe are formed and located within galaxies. However, Zwicky (1951)'s result implies that there are many luminous stars located between galaxies. A significant amount of stars must be far from galaxies, as implied by the brightness of the diffused light.

In order to further investigate this problem, over the years, there have been many more observations made about these inter-galactic objects. Red giants have been observed in a blank field in the Virgo Cluster as shown in Durrell et al. (2002). Planetary nebulae have been observed in the inter-galactic space of the M81 groups of galaxies (Feldmeier et al. 2004), and also in the Virgo Cluster (Ferguson et al. 1998, Arnaboldi et al. 2002) and Coma Cluster (Gerhard et al. 2005). Moreover, Gal-Yam et al. (2003) even found supernovae between galaxies.

Among these works, Durrell et al. (2002) suggested that intra-cluster stars could contribute $10 \% \sim 20 \%$ of the total I-band luminosity of the Virgo cluster. Furthermore, Mihos et al. (2005) recently observed Virgo cluster's core, as shown in their Figure 1 and 2, and found that there are a lot of intra-cluster stars and tidal tails between galaxies in the cluster.

With the above observational results, many interesting questions on the production of intergalactic populations could be asked. Did these stars form through the collapse of inter-galactic medium ? Are they the remnants of stellar groups during the early phase of the formation of a cluster of galaxies? Are they the outcome of continuous stripping due to the merging and interaction between galaxies? In order to obtain good answers, many theoretical studies on this subject have been done. For example, focusing on clusters of galaxies, cosmological n-body simulations have been employed to investigate the origins of intra-cluster populations (Please see Napolitano et al. 2003, Murante et al. 2004, Willman et al. 2004, Sommer-Larsen et al. 2005, Rudick et al. 2006, Murante et al. 2007). Using an observational definition of intra-cluster light (ICL) to be luminosity at a surface brightness $\mu_{V}>26.5$ mag $\operatorname{arcsec}^{-2}$, Rudick et al. (2006) found that about $10 \% \sim 15 \%$ of the clusters' luminosity is at ICL surface brightness. In their simulations, the tidal stripping of cluster galaxies could be one of the main mechanisms to produce ICL. However, Murante et al. (2007) concluded that the majority of the diffused stellar component is produced during the merging in the formation history of the bright cluster galaxies.

Although inter-galactic stellar populations and the ICL have been investigated by many groups both observationally and theoretically, as reviewed in Zibetti (2008), one of the main issues is about the very definition of inter-galactic stellar populations and the ICL. All photometric studies have to define criteria, such as surface brightness thresholds etc. to isolate the light of inter-galactic stars from the galactic signal. Note that the previously mentioned work on clusters of galaxies employed different working definitions of the ICL with their own chosen criteria. However, from the dynamical point of view, the light from the stars which are not bound to any galactic potential (with dark matter included) seems to be a fundamental choice.

On the other hand, due to that van Dokkum et al. (1999) presented many examples of mergers of E/S0, or early-type spiral galaxies, Stanghellini et al.(2006) went on to study the production of inter-galactic populations through the mergers of elliptical galaxies. In their work, a pair of elliptical galaxies with a given initial mass ratio and initial relative velocity, represented by spherical 
distributed N-body systems, is set to have a head-on collision. The massive halo of dark matter is not considered in the above simulations. They conclude that this scenario is helpful to feed $5 \% \sim 20 \%$ of the total initial mass as unbound stars.

The work in Stanghellini et al.(2006) presents a good example to study the production of gravitational unbound population (GUP) by a merging event. This types of work could compromise the cosmological n-body simulations on clusters, in the sense that (a) the detail relationship between GUP productions and the ways of merging could be investigated and (b) the members of GUP are well defined and easy to be identified in simulations. As Zibetti (2008) mentioned, in one particular system of galaxies, GUP shall be different from those photometric ICL, and to identify both of them observationally would be an important future work.

To take a further step from Stanghellini et al.(2006), we here study the merging events of spiral galaxies. The discs are used to represent the stellar components of spiral galaxies and there is no gaseous component. Thus, our model galaxies may be regarded as early-type spiral galaxies or late-type spiral galaxies in which the gaseous effect is ignored. Although ICL is our motivation for this work, we will only investigate the production of GUP here. The well-defined GUP could give strong constraint on the dynamical evolution of mergers.

Moreover, because dark matter is more massive than the stellar part (Faber 1987) and shall be dynamically important, we include the dark haloes in the systems. Indeed, the dark halo plays a crucial role in galactic dynamics. For example, Jiang \& Binney (1999) demonstrated that the infall of dark matter could influence the orientation of the dark halo, and then warp the stellar disc. Jiang \& Binney (2000) further showed that the dynamical friction from the Milky Way's dark halo could successfully explain the orbit of the Sagittarius dwarf galaxy.

\section{The Model}

In this project, we study the head-on mergers of spiral galaxies and their contributions on the production of GUP. A pair of galaxies with dark haloes will be employed for the simulations of merging events. Each galaxy includes two components, the stellar disc and the dark halo, with the density profiles as described in Hernquist (1993). These two components will be allowed to influence each other and relax to approach a new combined equilibrium. The parallelized version of the code, GADGET, is used for all N-body simulations (Springel et al. 2001), and the force softening is set as $0.075 \mathrm{kpc}$ for both the stellar disc and dark halo.

On the other hand, as shown in van Dokkum et al. (1999), there are many different possible relative angles between two discs in a merger. In order to study this effect, we choose six different relative orientations between the angular momentums of two discs. The galaxy located at $(x, y, z)=(0,-150,0)$ would always have an angular momentum with the direction $\hat{L}=(0,0,1)$, but the galaxy at $(x, y, z)=(0,150,0)$ would have an angular momentum with six different directions. For parabolic (hyperbolic) mergers, the galaxy at $(x, y, z)=(0,150,0)$ would have an angular momentum with direction $\hat{L_{1}}=(0,0,1)$ in Model P1 $(\mathrm{H} 1), \hat{L_{2}}=(0,-1,0)$ in Model P2 $(\mathrm{H} 2), \hat{L_{3}}=(0,0$, -1) in Model P3 (H3), $\hat{L_{4}}=(0,1,0)$ in Model P4 (H4), $\hat{L_{5}}=(1,0,0)$ in Model P5 (H5), $\hat{L_{6}}=(-1,0$, 0) in Model P6 (H6), respectively. The discs of two galaxies are said to be parallel in Model P1 (H1), anti-parallel in Model P3 (H3), and perpendicular in the rest models. Please see Wu \& Jiang (2009) for further details. 


\section{The Results}

We calculate the total energy of both stellar and dark-matter particles, in order to identify the GUP, and then determine their fractions. In Table 1, the first row gives the percentage of stellar GUP particles (compared to the total number of stellar particles) and the 2nd row lists the percentage of dark-matter GUP particles (compared to the total number of dark-matter particles) at the end of simulations in parabolic mergers. For the stellar GUP, the average percentage is $m_{P s}=0.1583$, and the standard deviation is $\sigma_{P S}=0.0524$. Almost all values are within or near the boundary of the interval $\left[m_{P s}-\sigma_{P s}, m_{P s}+\sigma_{P S}\right]=[0.1059,0.2107]$. For the dark-matter GUP, the mean is $m_{P d}=7.7883$ with a standard deviation $\sigma_{P d}=0.0508$. The interval $\left[m_{P d}-\sigma_{P d}, m_{P d}+\sigma_{P d}\right]=$ $[7.7375,7.8391]$ Only the dark-matter GUP of Model P6 is a bit smaller and has a value about $m_{P d}-2 \sigma_{P d}$.

\begin{tabular}{|l||c|c|c|c|c|c|}
\hline Model & P1 & P2 & P3 & P4 & P5 & P6 \\
\hline \hline stellar GUP (\%) & 0.16 & 0.10 & 0.18 & 0.08 & 0.22 & 0.21 \\
\hline dark-matter GUP $(\%)$ & 7.84 & 7.80 & 7.83 & 7.76 & 7.81 & 7.69 \\
\hline
\end{tabular}

Table 1: The percentages of stellar GUP and dark-matter GUP in parabolic mergers.

In Table 2, we list the percentages of stellar GUP (the first row) and dark-matter GUP (the second row) at the end of simulations in hyperbolic mergers. For the stellar GUP, the average percentage is $m_{H s}=0.3183$, and the standard deviation is $\sigma_{H s}=0.0809$. The first interval $\left[m_{H s}-\sigma_{H s}, m_{H s}+\sigma_{H s}\right]=[0.2374,0.3992]$, and the second interval $\left[m_{H s}-2 \sigma_{H s}, m_{H s}+2 \sigma_{H s}\right]=$ $[0.1565,0.4801]$. The stellar GUPs of Model H1, H5, H6 are within the first interval, and the ones of Model H2, H3, H4 are within the second interval. For the dark-matter GUP, the mean is $m_{H d}=14.1517$ with a standard deviation $\sigma_{H d}=0.1446$. The $1 \sigma$ interval $\left[m_{H d}-\sigma_{H d}, m_{H d}+\right.$ $\left.\sigma_{H d}\right]=[14.0071,14.2963]$ and $2 \sigma$ interval $\left[m_{H d}-2 \sigma_{H d}, m_{H d}+2 \sigma_{H d}\right]=[13.8625,14.4409]$. Thus, the values of Model H1, H2, H5, H6 are in $1 \sigma$ interval, and the ones of Model H3, H4 are within $2 \sigma$ interval approximately.

\begin{tabular}{|l||c|c|c|c|c|c|}
\hline Model & H1 & H2 & H3 & H4 & H5 & H6 \\
\hline \hline stellar GUP $(\%)$ & 0.37 & 0.21 & 0.42 & 0.21 & 0.33 & 0.37 \\
\hline dark-matter GUP $(\%)$ & 14.22 & 14.12 & 14.33 & 13.86 & 14.19 & 14.19 \\
\hline
\end{tabular}

Table 2: The percentages of stellar GUP and dark-matter GUP in hyperbolic mergers.

\section{Conclusions}

In order to investigate the relationship between the galactic mergers and GUP, we have used the combined disc-halo systems to model the head-on merging events of spiral galaxies. We consider both the parabolic and hyperbolic mergers, and we propose six models for each of the mergers, with different relative orientations between two merging galaxies. 
We find that the timing of the merging process does not depend on the relative orientation. Nevertheless, the resulting structures of stellar components show a great variety for different disc orientations.

In terms of the production of GUP, we found that head-on merging events produce much more dark-matter GUP than the stellar GUP. This could be due to that the stellar particles are initially located at the very central part of whole systems, and thus more difficult to become gravitational unbound. On the other hand, the dark-matter particles distribute over a large range, and those initially located at the outer part could be striped out of the system easily.

Moreover, due to the higher energy injection rate of the hyperbolic merging process, it produces more GUP than the parabolic one (about a factor of two). We also found that, in Model P1-P6, the merging event starts to produce GUP quickly at the time $t=8 T_{d y n}$ ( $T_{d y n}$ is the dynamical time), which is actually the time when the first encounter happens. After $t=10 T_{d y n}$, very little stellar GUP particles are produced. In Model H1-H6, the production starts at $t=5 T_{d y n}$. The production rate is very high from $t=5 T_{d y n}$ to $t=6 T_{d y n}$. Then, the stellar GUP slowly increases until $t=15 T_{d y n}$. Therefore, the energy injection rate is essential for the production of GUPs.

On the other hand, we also calculated the mean and standard deviation of GUP percentages. For parabolic mergers, the GUP percentages are all within or close to the boundary of $1 \sigma$ interval. For hyperbolic mergers, some model's GUP percentages are in $1 \sigma$ interval but some others are inside or near the boundary of $2 \sigma$ interval. Therefore, in addition to producing more GUP, the hyperbolic mergers also show larger scattering in terms of GUP production.

In general, our results show that, depending on the relative orientation and the relative velocity, a head-on collision of a galaxy pair (with dark matter) would make less than one percent of the initial stellar mass become the luminous GUP but about eight to fourteen percent of the dark matter become the dark GUP. Therefore, multiple mergers are needed to produce much more stellar GUPs.

\section{References}

[1] Arnaboldi, M., Aguerri, J. A. L., Napolitano, N. R., Gerhard, O., Freeman, K. C., Feldmeier, J., Capaccioli, M., Kudritzki, R. P., Mendez, R. H., 2002, AJ, 123, 760

[2] Durrell, P. R., Ciardullo, R., Feldmeier, J. J., Jacoby G. H., \& Sigurdsson, S., 2002, ApJ, 570, 119

[3] Faber, S. M., 1987, Nearly Normal Galaxies: From the Planck Time to the Present; Proceedings of the Eighth Santa Cruz Summer Workshop in Astronomy and Astrophysics, Springer-Verlag, New York

[4] Feldmeier, J. J., Ciardullo, R., Jacoby, G. H., Durrell, P. R., 2004, ApJ, 615, 196

[5] Ferguson, H. C., Tanvir, N. R., von Hippel, T., 1998, Nature, 391, 461

[6] Gal-Yam, A., Maoz, D., Guhathakurta, P., Filippenko, A. V., 2003, AJ, 125, 1087

[7] Gerhard, O., Arnaboldi, M., Freeman, K. C., Kashikawa, N., Okamura, S., Yasuda, N., 2005, ApJ, 621, L93

[8] Hernquist, L., 1993, ApJS, 86, 389

[9] Jiang, I.-G., Binney, J., 1999, MNRAS, 303, L7

[10] Jiang, I.-G., Binney, J., 2000, MNRAS, 314, 468

[11] Mihos, J. C., Harding, P., Feldmeier, J., \& Morrison, H., 2005, ApJ, 631, L41 
[12] Murante, G. et al., 2004, ApJ, 607, L83

[13] Murante, G. et al., 2007, MNRAS, 377, 2

[14] Napolitano, N. R. et al., 2003, ApJ, 594, 172

[15] Rudick, C. S., Mihos, J. C., McBride, C., 2006, ApJ, 648, 936

[16] Sommer-Larsen, J., Romeo, A. D., Portinari, L., 2005, MNRAS, 357, 478

[17] Springel, V., Yoshida, N., \& White, S.D.M., 2001, NewA, 6, 79

[18] Stanghellini, L., Gonzalez-Garcia, A. C., \& Manchado, A., 2006, ApJ, 644, 843

[19] van Dokkum, P. G., Franx, M., Fabricant, D., Kelson, D. D., Illingworth, G. D., 1999, ApJ, 520, L95

[20] Willman, B., Governato, F., Wadsley, J., Quinn, T., 2004, MNRAS, 355, 159

[21] Wu, Y.-T., Jiang, I.-G., 2009, MNRAS, 399, 628

[22] Zibetti, S., 2008, Proceedings of the IAU Symposium, 244, 176

[23] Zwicky, F., 1951, PASP, 63, 61 